\title{
Facteurs De Retard Diagnostique De La Tuberculose Pulmonaire Vus À l'Unité De Soins, De Formation Et De Recherche De Pneumologie Befelatanana
}

\author{
Ravahatra Kiady, \\ Michel Tiaray Harison, \\ Chef de Clinique en Pneumologie, Faculté de Médecine d'Antananarivo, \\ Université d'Antananarivo, Madagascar \\ Rakotondrabe Iantsotiana Davidson, \\ Rasoafaranirina Marie Odette, \\ Nandimbiniaina Anjara,
} Interne des Hôpitaux en Pneumologie, Faculté de Médecine d'Antananarivo, Université d'Antananarivo, Madagascar

Rakotomizao Jocelyn Robert,

Chef de Service de Pneumologie, Faculté De Médecine Université d'Antananarivo, Madagascar

Rakotoson Joelson Lovaniaina,

Professeur Agrégé en Pneumologie, Faculté de Médecine de Fianarantsoa,

Université de Fianarantsoa, Madagascar

Raharimanana Rondro Nirina

Professeur Titulaire en Pneumologie, Faculté De Médecine Université

d'Antananarivo, Madagascar

doi: 10.19044/esj.2017.v13n27p413 URL:http://dx.doi.org/10.19044/esj.2017.v13n27p413

\begin{abstract}
Delay in diagnosis is significant to tuberculosis prognosis. The aim of this study is to evaluate the diagnosis delay of tuberculosis and to identify determinants of "patient delay", "health system delay", and "total delay" in the diagnosis of tuberculosis. It is a prospective study of the patients who were hospitalized at the Unité de Soins de Formation et de Recherche (USFR) of Pneumology in Befelatanana, Antananarivo Madagascar, during the period of $1^{\text {st }}$ October 2014 to 30 April 2015 (7 months). We included all patients with diagnosis of pulmonary tuberculosis. Sixty six patients were also included. The mean time of patient delay, health system delay, and total delay were $26,30 \pm 36,87,69,56 \pm 64$, and $96,35 \pm 72,65$ days respectively. The different variables that affected diagnosis delay were: tobacco smoke (OR : 3,6723), asthenia (OR : 5,4815), anorexia (OR : 2,9524), and hemoptysis
\end{abstract}


(OR : 0,2406) for the total delay. Knowledge about tuberculosis signs (OR : $0,164)$ and transmissions (OR : 0,243) was for the patient delay. Hemoptysis (OR : 8,1250), asthenia (OR : 0,1081), breathlessness (OR : 0,3556), infiltrative syndrome (OR : 0,2500), and alveolar syndrome (OR : 0,0687) in chest Xray was for the health system delay. Having an understanding of these factors of tuberculosis diagnosis delay will result to a decrease in the diagnosis delay.

Keywords: Delay, tuberculosis, diagnosis, patient, health system, Antananarivo, Madagascar

\section{Résumé}

Le délai de prise en charge conditionne l'évolution et le pronostic de la tuberculose, Cette étude a pour objectifs d'évaluer le délai diagnostique de la tuberculose et d'identifier les facteurs de retard diagnostique pour le «délai du patient», le «délai du système de soins» et le «délai total». C'est une étude prospective des patients hospitalisés à l'Unité de Soins, de Formation et de Recherche (USFR) de Pneumologie du Befalatanana, Antananarivo Madagascar pendant la période du $1^{\mathrm{er}}$ octobre 2014 au 30 avril 2015 (7 mois). Ont été inclus, les patients présentant un diagnostic de tuberculose pulmonaire. Soixante-six patients ont été inclus. Les moyennes du délai patient, délai système de soins et délai total étaient respectivement de $26,30 \pm 36,87,69,56 \pm 64$, et $96,35 \pm 72,65$ jours. Les différentes variables affectant les délais étaient : le tabagisme (OR : 3,6723), l'asthénie (OR : 5,4815), l'anorexie (OR: 2,9524), l'hémoptysie (OR: 0,2406), l'expectoration (OR : 16,9218) pour le délai total. L'habitat (OR : 5,203), la connaissance des signes (OR : 0,164) et le mode de contamination (OR : 0,243 ) pour le délai patient, hémoptysie (OR : 8,1250), asthénie (OR : 0,1081), dyspnée (OR:0,3556), la présence d'un syndrome infiltratif $(\mathrm{OR}: 0,2500)$ et de syndrome alvéolaire $(\mathrm{OR}: 0,0687)$ à la radiographie du thorax pour le délai système de soins. La connaissance de ces facteurs de retard diagnostique de la tuberculose va permettre de mener une action pour diminuer le délai diagnostique.

Mots-clés: Délai, tuberculose, diagnostic, patient, système de soins, Antananarivo, Madagascar

\section{Introduction}

La tuberculose est l'une des causes de morbidité et de mortalité les plus fréquentes au monde, on estime à 8,8 millions le nombre de nouveaux cas par an et à plus de 1,4 million le nombre de décès dû à cette maladie. 
Plus de 95\% de la mortalité et de la morbidité de la tuberculose s'observent dans les pays en développement (WHO, 2012).

Dans notre pays, on trouve toujours des formes graves liées au retard diagnostique qui peuvent mettre en jeu le pronostic vital du patient ou laisser des séquelles importantes et contribuent également à la diffusion de l'épidémie. En effet un patient tuberculeux contagieux peut infecter 10 à 20 personnes au cours de l'histoire naturelle de sa maladie dans les pays en voie de développement (Lawn, 1998). La maîtrise de cette épidémie ne peut se réaliser qu'en faisant un diagnostic précoce, limitant ainsi la transmission du bacille. L'objectif de notre étude est de rechercher les principaux facteurs de retard de diagnostic de la tuberculose pulmonaire chez les patients tuberculeux vus à l'Unité de Soins, de Formations et de Recherches (USFR) de Pneumologie de Befelatanana, Antananarivo. Cette étude permettrait de pallier ce retard de diagnostic afin de bien maitriser ce fléau et de rendre plus efficace le programme de lutte contre la tuberculose.

\section{Méthodes}

Il s'agit d'une étude prospective, descriptive et analytique réalisée dans le service de Pneumologie à l'hôpital Befelatanana, Antananarivo Madagascar pendant la période du 1 ${ }^{\mathrm{er}}$ octobre 2014 au 30 avril 2015 (7 mois).

Nous avons inclus les patients hospitalisés pendant la période d'étude et présentant un diagnostic de tuberculose pulmonaire.

Nous avons exclus tous les patients qui n'ont pas la possibilité à s'exprimer (trouble de la conscience) et les patients incapables de répondre au questionnaire.

La première étape de l'étude consistait à l'établissement des questionnaires de recueil des données après une revue bibliographique.

La deuxième étape de l'étude consistait à remplir les questionnaires en faisant une interrogatoire des patients dès que le diagnostic d'une tuberculose pulmonaire a été posé. définis:

Au cours de notre étude, les délais diagnostiques suivants ont été

- Délai du patient: C'est le délai qui s'écoule entre l'apparition des premiers symptômes à la première consultation chez un prestataire de soins. Il a été considéré comme long lorsqu'il dépasse 30 jours.

- Délai du système de soins: C'est le délai entre la première consultation chez un professionnel de santé jusqu'à l'initiation du traitement. Il a été considéré comme long lorsqu'il dépasse 30 jours.

- Délai total: C'est le délai qui s'écoule entre l'apparition des premiers signes jusqu'à l'initiation du traitement. C'est-à-dire le résultat de 
la somme de ces deux délais cité en haut. Il a été considéré comme long lorsqu'il dépasse 60 jours.

Le niveau d'instruction a été classé comme suit :

- $\quad$ Primaire : classe de $12^{\text {ème }}$ jusqu'au classe de $7^{\text {ème }}$

- $\quad$ Secondaire niveau I : classe de 6ème jusqu' au classe de 3ème

- Secondaire niveau II : classe de seconde jusqu'au classe de terminale

- Universitaire : toutes les études effectuées après le Baccalauréat

Nous avons considéré que les patients connaissent les symptômes de la tuberculose lorsqu'ils répondent par « toux chronique ». Nous avons considéré que les patients connaissent le mode de contamination de la tuberculose lorsqu'ils répondent «par la toux ». Nous avons demandé aux patients d'estimer le nombre de jours écoulés entre le début des symptômes et le recours aux soins médicaux.

Les symptômes les plus fréquemment associés avec la tuberculose ont été classés comme dus à la tuberculose et comprennent anorexie, douleurs thoraciques, frissons, toux, fatigue, fièvre, hémoptysies, sueurs nocturnes et perte de poids. Tous les autres symptômes moins fréquemment associés avec la tuberculose ont été classés comme non tuberculeux.

Les données ont été saisies sur le logiciel EXCEL et les analyses des données ont été effectuées sur le logiciel EPI-INFO. Le test de CHI2 a été utilisé pour la comparaison des données, et le test de régression logistique pour l'obtention de l'odds ratio (OR). Nous avons pris comme seuil de significativité $\mathrm{p}<0,05$.

\section{Résultats}

Durant la période d'étude, 75 patients présentaient une tuberculose pulmonaire soit $12,21 \%$ des patients hospitalisés et $81,5 \%$ des tuberculoses toutes formes confondues.

Neuf patients étaient exclus devant leurs incapacités à répondre au questionnaire et leur non coopération. Soixante-six patients (88\%) ayant répondu aux critères d'inclusion et d'exclusion ont été retenus. Parmi ces cas, 5 cas $(7,58 \%)$ étaient une tuberculose pulmonaire à bacilloscopie négative (TPM-), et 61 cas $(92,42 \%)$ étaient une tuberculose pulmonaire à bacilloscopie positive (TPM+).

Les moyennes du délai patient, délai système de soin et délai total étaient respectivement de $26,30 \pm 36,87$ jours, $69,56 \pm 64,10$ jours et 96,45 $\pm 72,65$ jours.

Trente-quatre patients $(51,51 \%)$ consultaient en premier lieu un médecin généraliste libéral, 12 patients $(18,19 \%)$ consultaient un médecin généraliste fonctionnaire, 8 patients $(12,12 \%)$ consultaient un guérisseur et 5 patients $(7,58 \%)$ ont fait une auto-médication. 
Les caractéristiques démographique, clinique et paraclinique des patients sont résumés dans le Tableau I et II et les facteurs associés au retard diagnostique de la tuberculose sont présentés dans le Tableau III.

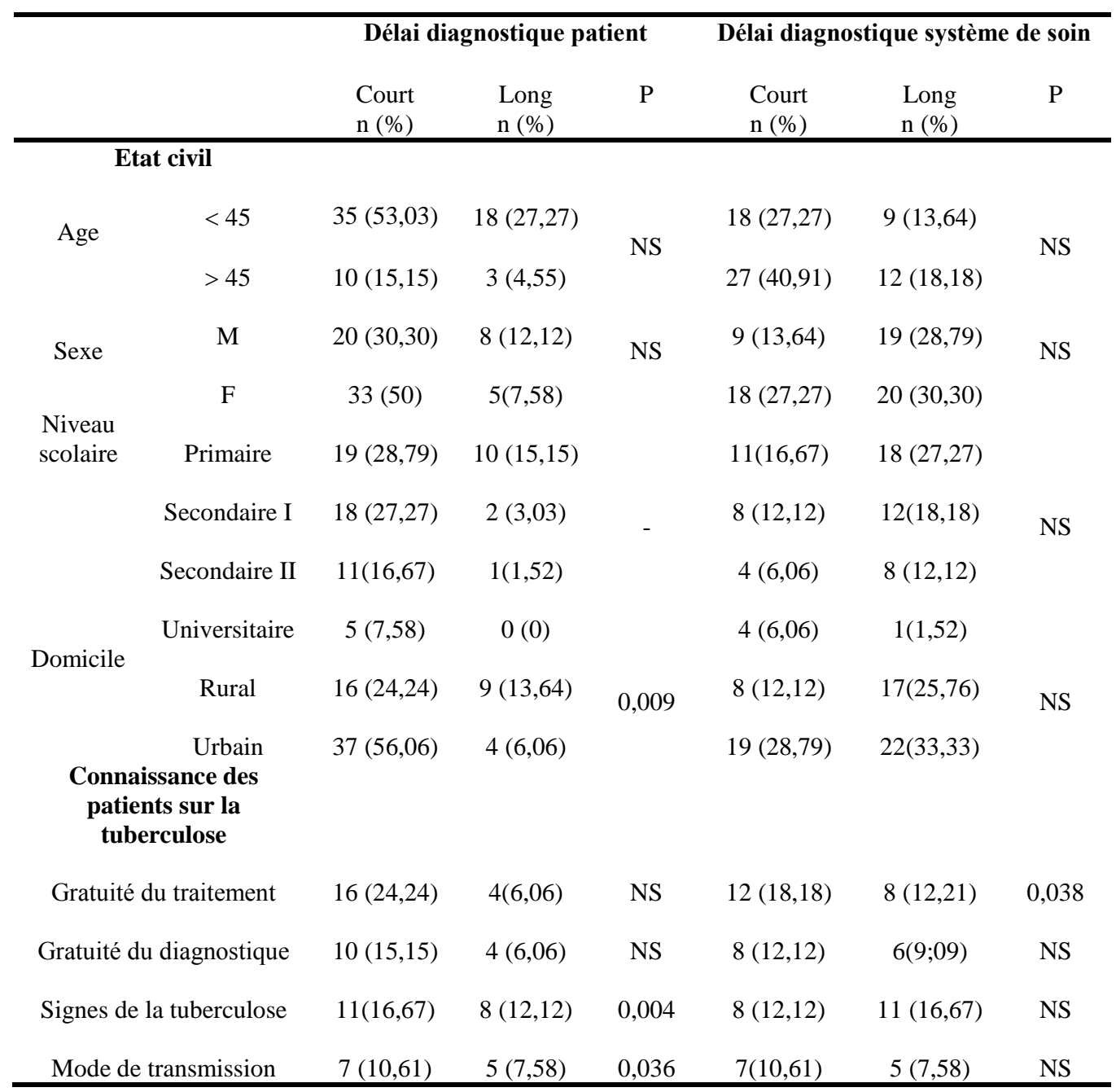

Tableau 1. Répartition de l'état civil et des connaissances des patients sur la tuberculose selon le délai diagnostique

NS : non significatif 


\begin{tabular}{|c|c|c|c|c|c|c|}
\hline & \multicolumn{3}{|c|}{ Délai diagnostique système de soin } & \multicolumn{3}{|c|}{ Délai diagnostique total } \\
\hline & $\begin{array}{l}\text { Court } \\
\mathrm{n}(\%) \\
\end{array}$ & $\begin{array}{l}\text { Long } \\
\mathrm{n}(\%)\end{array}$ & $\mathrm{P}$ & $\begin{array}{l}\text { Court } \\
\mathrm{n}(\%) \\
\end{array}$ & $\begin{array}{l}\text { Long } \\
\mathrm{n}(\%)\end{array}$ & $\mathrm{P}$ \\
\hline \multicolumn{7}{|l|}{ Antécédent } \\
\hline Tabagisme & $10(15,15)$ & $20(30,30)$ & NS & $7(10,61)$ & $23(34,85)$ & 0,015 \\
\hline \multicolumn{7}{|l|}{ Signes généraux } \\
\hline Asthénie & $18(27,27)$ & $37(56,06)$ & 0,003 & $18(27,27)$ & $37(56,06)$ & 0,013 \\
\hline Anorexie & $15(22,73)$ & $30(45,45)$ & NS & $14(21,21)$ & $31(46,97)$ & 0,013 \\
\hline Fièvre & $21(31,82)$ & $34(51,52)$ & NS & $21(31,82)$ & $34(51,52)$ & NS \\
\hline Amaigrissement & $22(33,33)$ & $37(56,06)$ & NS & $21(31,82)$ & $38(57,58)$ & NS \\
\hline \multicolumn{7}{|l|}{ Signes cliniques } \\
\hline Toux & $27(40,91)$ & $39(59,09)$ & NS & $26(39,39)$ & $40(60,61)$ & NS \\
\hline \multirow{2}{*}{$\begin{array}{c}\text { Hémoptysie } \\
\text { Douleur thoracique }\end{array}$} & $13(19,70)$ & $4(6,06)$ & 0,001 & $11(16,67)$ & $6(9,09)$ & 0,013 \\
\hline & $7(10,61)$ & $15(22,73)$ & NS & $7(10,61)$ & $15(22,73)$ & NS \\
\hline Dyspnée & $12(18,18)$ & $27(40,91)$ & 0,044 & $12(18,18)$ & $27(40,91)$ & NS \\
\hline \multicolumn{7}{|l|}{$\begin{array}{c}\text { Signes } \\
\text { radiologiques }\end{array}$} \\
\hline Excavation & $15(22,73)$ & $19(28,79)$ & NS & & - & \\
\hline Infiltrative & $17(25,76)$ & $34(51,52)$ & 0,021 & - & - & \\
\hline Alveolaire & $1(1,52)$ & $14(21,21)$ & 0,021 & & & \\
\hline Nodule & $0(0)$ & $0(0)$ & - & & - & \\
\hline Pleurésie & $1(1,52)$ & $2(3,03)$ & NS & - & - & \\
\hline
\end{tabular}

Tableau 2. Répartition des manifestations cliniques et radiologiques selon le délai diagnostique

NS : non significatif 


\begin{tabular}{|c|c|c|c|c|}
\hline & Variable & $\begin{array}{l}\text { Odds } \\
\text { Ratio } \\
\text { (OR) } \\
\end{array}$ & $\begin{array}{c}\text { Intervalle de } \\
\text { confiance } 95 \%\end{array}$ & $\mathbf{p}$ \\
\hline \multirow[t]{3}{*}{ Délai patient } & Habitat & 5,203 & $(1,396-19,394)$ & 0,009 \\
\hline & $\begin{array}{c}\text { Connaissance des signes de } \\
\text { la tuberculose par les } \\
\text { patients }\end{array}$ & 0,164 & $(0,045-0,600)$ & 0,004 \\
\hline & $\begin{array}{l}\text { Connaissance de la mode } \\
\text { de contamination par les } \\
\text { patients }\end{array}$ & 0,243 & $(0,062-0,960)$ & 0,004 \\
\hline \multirow{5}{*}{$\begin{array}{l}\text { Délai système de } \\
\text { soin }\end{array}$} & Asthénie & 0.1081 & $(0.0211-0.5531)$ & 0.0076 \\
\hline & Hémoptysie & 8.1250 & $(2.2585-29.2296)$ & 0.0013 \\
\hline & dyspnée & 0,3556 & $(0,1283-0,9852)$ & 0,0467 \\
\hline & Syndrome infiltratif & 0.2500 & $(0.0737-0.8478)$ & 0.0261 \\
\hline & Syndrome alvéolaire & 0.0687 & $(0.0084-0.5617)$ & 0.0125 \\
\hline \multirow[t]{5}{*}{ Délai total } & Tabac & 3,6723 & $(1,2606-10,6977)$ & 0,0171 \\
\hline & Asthénie & 5,4815 & $(1,2968-23,1699)$ & 0,0207 \\
\hline & Anorexie & 2,9524 & $(1,0125-8,6087)$ & 0,0474 \\
\hline & Hémoptysie & 0.2406 & $(0.0750-0.7719)$ & 0,0166 \\
\hline & Expectoration & 16,9218 & $(1,6746-170,9912)$ & 0,0165 \\
\hline
\end{tabular}

Tableau 2. Facteur déterminant des retards diagnostique pour le délai patient, système de soins et le délai total NS : non significatif

\section{Discussion}

Les retards dans le diagnostic de la tuberculose peuvent avoir pour origine le patient ou le système de santé. Dans notre étude les moyennes du délai patient, délai institution et délai total étaient respectivement de $26,30 \pm 36,87$ jours, $69,56 \pm 64$ jours, et $96,35 \pm 72,65$ jours. Notre délai patient était plus court que celui de 7 à 8 semaines rapportées dans des pays plus développés (Aoki, 1985; Mori, 1992) et dans la plupart des pays d'Afrique : 89,6 jours en Ghana (Lawn, 1998), 179 jours en Éthiopie (Demissie, 2002), tandis qu'un délai patient plus court a été rapporté dans la région sud de l'Inde (20 jours) (Rajeswari, 2002). Selon l'auteur, ce délai court est dû à la prévalence élevée de la tuberculose qui entrainait une vigilance élevée de la part des médecins quant au diagnostic de la tuberculose. Par ailleurs, la valeur médiane du retard de système de soins à 69 jours était longue par rapport à une valeur de 36 jours pour Kathmandu Valley (Karki, 2004) et de 33 jours pour la partie sud de l'Inde (Rajeswari, 2002). Un délai système plus court a été retrouvé en Éthiopie (6 jours) (Demissie, 2002).

Actuellement, il n'y a pas de consensus sur le délai diagnostique acceptable de la tuberculose. Il est généralement considéré que pour un contrôle efficace de la tuberculose, le délai patient ne doit pas dépasser de 2 
à 3 semaines, le délai total ne doit pas dépasser de 3 à 4 semaines. Les délais trouvés dans notre étude avaient largement dépassé ce délai diagnostique acceptable. Cela peut être dû au fait que, la majorité des patients perçoit leur maladie comme étant une affection sans gravité et a une toux banale souvent négligée, mais aussi au méconnaissance des signes cliniques de la tuberculose de la part des praticiens et leurs difficultés à entreprendre une action rapide pour prescrire des investigations adaptées soit d'orienter le patient vers les centres de diagnostic publics.

Dans notre étude, l'habitat des patients était un déterminant du délai patient pour le diagnostic de la tuberculose. Les patients vivant en milieu rural étaient associés à un retard diagnostique par rapport aux patients vivant en milieu urbain. Deux études éthiopiennes ont noté une association entre une résidence en zone rurale et l'allongement du délai du patient avec des forces d'association comprise entre 1,4 et 2,4 (Cambanis, 2015; Mesfin, 2009). Plusieurs autres études ont aussi montré le rôle de la ruralité dans le retard diagnostique de la tuberculose (Lawn, 1998; Karki, 2004). Une telle situation peut s'expliquer par la précarité plus grande des populations rurales, la difficulté d'accès au centre de santé et/ou par une couverture géographique sanitaire insuffisante. Les patients passent beaucoup de temps pour venir au centre de santé (le plus souvent à pied), ce qui est encore plus fatigant pour les patients et favorise le retard diagnostique. Les patients vivant en zone rurale ont aussi une faible connaissance sur la tuberculose. Par contre dans une étude réalisée à New York, une villes développé, les retards ne sont pas associés avec le lieu de résidence, ce qui est peut être dû à la bonne accessibilité physique et la disponibilité des soins à New York (Sherlan, 1999).

Concernant l'antécédent des patients, seule la présence d'un antécédent de tabagisme était associée à un allongement de délai patient. Plusieurs études dans la littérature ont confirmé cette association avec des odds ratio de 1,6 à 3,7. Les personnes qui fument plus de 5 cigarettes par jour étaient significativement associées à un haut risque de retard diagnostique. Cette association semble être dose dépendante, les gens qui fument moins présentent moins de risque de retard que les gens qui fument beaucoup plus. Mais, quel que soit le nombre de tiges, les fumeurs présentent un risque élevé de retard diagnostique par rapport au non-fumeur (Basnet, 2009). Dans la vie quotidienne, les gens qui fument ont l'habitude d'avoir des toux chroniques (signe de bronchite chronique). Ces signes vont être confondus avec les signes de la tuberculose et ces gens ne vont consulter que lorsque d'autres signes gênants vont apparaitre ou lorsque leur état va s'aggraver (Basnet, 2009; Woith, 2008). En plus les personnels de santé peuvent aussi penser que la toux est associée au tabagisme et ne vont pas penser à la tuberculose. 
Dans notre étude, la connaissance des signes de la tuberculose et du mode de contamination par le patient raccourcissait le délai de la première consultation avec respectivement un Odds Ratio de 0,164 (0,045 - 0,600), $0,243(0,062-0,960)$. La méconnaissance des symptômes et du mode de transmission de la tuberculose et/ou de son étiologie a été associé à un allongement du temps du patient dans plusieurs études ( $\mathrm{Wu}, 2008$; Zou, 2009; Li, 2002), tandis qu'à l'inverse, Wang et al ont montré en Chine qu'une bonne connaissance de la tuberculose peut contribuer à la réduction du délai patient avec un odds ratio de 0,63 (Wang, 2008).

Dans notre étude, un allongement du délai total du diagnostic était associé à la présence d'asthénie et d'anorexie avec respectivement un Odds Ratio de 5,4815 (1,2968 -23,1699), 2,9524 (1,0125-8,6087). Par contre, le délai système de soins était raccourci devant une asthénie avec un Odds Ratio de 0.1081 (0.0211-0.5531). Nous n'avons noté qu'une seule étude qui a montré l'impact des signes généraux sur le retard diagnostique de la tuberculose. Cette étude a trouvé que la présence d'une perte de poids était associée au retard diagnostique de la tuberculose. L'explication était que la perte de poids met beaucoup plus de temps pour être remarquée par les patients (Aker, 2011). Pour notre étude, cela peut être dû au fait que ces signes sont non spécifiques de la tuberculose, il existe de nombreuses pathologies qui peuvent donner les mêmes symptomatologies. Les médecins praticiens ne pensent pas systématiquement au diagnostic de la tuberculose devant ces signes non spécifiques. Par ailleurs, ils sont souvent négligés par les patients et qui vont attendre les complications avant de consulter un médecin. La présence de ces signes traduit déjà un état avancé de la maladie.

Concernant les signes cliniques, la présence d'hémoptysie est un facteur raccourcissant le délai total $(\mathrm{OR}=0.2406$, IC : 0.0750-0.7719), mais paradoxalement c'est un facteur allongeant le délai système de soins (OR : 8.1250 , IC : 2.2585-29.2296). La présence de la dyspnée est un facteur raccourcissant le délai système de soins (OR : 0.3556, IC : 0.1283-0.9852). Dans la littérature la présence d'une hémoptysie réduit le délai patient avec des forces d'association compris entre 1,11 et 25,29 (Godfrey, 2002; Rojpibulstit, 2006; Xu, 2005). En revanche, les signes peu spécifiques tels que la toux, l'expectoration et les sueurs nocturnes semblent avoir eu un effet inverse sur le délai du patient. Cette situation peut être due à une méconnaissance des symptômes de la tuberculose par les malades ou l'existence de signes chroniques semblables à ceux-ci. Seule une étude réalisée en Chine a observé qu'un signe typique comme l'hémoptysie multipliait par dix le risque d'allongement du délai du système de soins $(\mathrm{Xu}$, 2005).

Nous avons aussi constaté dans notre étude que la présence d'un syndrome infiltratif et alvéolaire radiologique était un facteur raccourcissant 
le délai système de soins avec un Odds ratio respectif de $0.2500(0.0737$ $0.8478), 0.0687$ (0.0084-0.5617). Nous n'avons pas trouvé d'étude qui a rapporté la relation entre les anomalies radiologiques et le retard diagnostique de la tuberculose. La présence d'une anomalie radiologique, quel qu'il soit permet au médecin de penser à la tuberculose. Dans notre pays, quand les médecins trouvent une anomalie radiologique qui persiste pendant longtemps, ils ont tendance à le traiter comme une tuberculose même si la recherche de BAAR dans le crachat est négative.

\section{Conclusion}

La tuberculose reste encore un problème de santé publique majeur dans notre pays. La connaissance des facteurs de retard diagnostique permet de limiter la transmission de cette pathologie. D'après les résultats de notre étude la réduction du délai patient est basée essentiellement sur l'éducation sanitaire. Elle vise à éclairer le public en particulier sur le mode et les causes favorisantes de la contamination et du développement de la maladie, sur les signes révélateurs de la tuberculose et sur la nécessité de la consultation précoce. La réduction du délai institution passe obligatoirement par l'amélioration du diagnostic et de la connaissance de la tuberculose par les médecins de première ligne, par l'intermédiaire d'une formation continue.

\section{References:}

1. Aker, SGH., Madland, S., Ullenes, M., Enarson, D., Rusen, ID., \& Kamara, D. (2011). Treatment delay among tuberculosis patients in Tanzania: Data from the FIDELIS Initiative. BMC Public Health; 11: 306.

2. Aoki, M., Mori, T., \& Shimao, T. (1985). Studies on factors influencing patient's, doctor's and total delay of tuberculosis casefinding in Japan. Bull Int Union Tuberc;60:128-30.

3. Basnet, R., Hinderaker, SG., Enarson, D., Malla, P., \& Morkve, O. (2009). Delay in the diagnosis of tuberculosis in Nepal. BMC Public Health; 9:236.

4. Cambanis, A., Yassin, MA., Ramsay, A., Squire, SB., Arbide, I., \& Cuevas, LE. (2015). Rural poverty and delayed presentation to tuberculosis services in Ethio-pia. Trop Med Int Health; 10(4):330-5

5. Demissie, M., Lindtjorn, B., \& Berhane, Y. (2002). Patient and health service delay in the diagnosis of pulmonary tuberculosis in Ethiopia. BMC Public Health;2:23.

6. Godfrey, RF., Kaunda, H., Kamanga, J., Beers, Sv., van Cleeff, M., Kumwenda-Phiri, R., et al. (2002). Why do patients with a cough delay seeking care at Lusaka urban health centres? A health systems research approach. Int J Tuberc Lung Dis; 6(9): 796-805. 
7. Karki, DK. (2004). Delay in Tuberculosis Treatment in Kathmandu Valley, Nepal. Thesis Tribhuvan University

8. Lawn, SD., Afful, B., \& Acheampong, JW. (1998). Pulmonary tuberculosis : diagnostic delay in Ghanaian adults. Int $\mathbf{J}$ Tuberc Lung Dis;2(8):635-40.

9. Li, AX., \& Wang, YL. (2002). Investigation of diagnostic delay of TB in Shang Qiu city. Henan Journal of Preventive Med; 13:292.

10. Mesfin, MM., Newell, JN., Walley, JD., Gessessew, A., \& Madeley, RJ. (2009). Delayed consultation among pulmonary tuberculosis patients: a cross sectional study of 10 DOTS districts of Ethiopia. BMC Public Health.;9:53.

11. Mori, T., Shimao, T., Jin, BW., \& Kim, SJ. (1992). Analysis of casefinding process of tuberculosis in Korea. Tubercle Lung Dis;73(4):225-31.

12. Rajeswari, R., Chandrasekaran, V., Suhadev, M., Sivasubramaniam, S., Sudha, G., \& Renu G. (2002). Factors associated with patient and health system delays in the diagnosis of tuberculosis in South India. Int J Tuberc Lung Dis;6(8):789-95.

13. Rojpibulstit, M., Kanjanakiritamrong, J., \& Chongsuvivatwong, V. (2006). Patient and health system delays in the diagnosis of tuberculosis in Southern Thailand after health care reform. Int $\mathbf{J}$ Tuberc Lung Dis;10(4):422-8.

14. Sherman, LF., Fujiwara, PI., Cook, SV., Bazerman, LB., \& Frieden, TR. (1999). Retards du patient et du système de santé dans le diagnostic et le traitement de la tuberculose. Int $\mathbf{J}$ Tuberc Lung Dis;3(12):1088-95.

15. Wang, Y., Long, Q., Liu, Q., Tolhurst, R., \& Tang, S. (2008). Treatment seeking for symptoms suggestive of TB: comparison between migrants and permanent urban residents in Chongqing, China. Trop Med Int Health;13(7):927-33.

16. Woith, WM., \& Larson, J. (2008). Delay in seeking treatment and adherence to tuberculosis medications in Russia: A survey of patients from two clinics. Int J Nurs Stud; 45(8): 1163-74.

17. World Health Organisation (2012). WHO report Global tuberculosis control.

http://apps.who.int/iris/bitstream/10665/75938/1/9789241564502_en g.pdf

18. Wu, ZJ., Zeng, RY., Shen, LT., \& Zhang, JF. (2008). Delay in identification of PTB in Shang Yang city. Pract Prev Med; 15: 448-9.

19. Xu, B., Jiang, QW., Xiu, Y., \& Kdiwan, V. (2005). Diagnostic delays in access to tuberculosis care in counties with or without the National 
Tuberculosis Control Programme in rural China. Int $\mathbf{J}$ Tuberc Lung Dis;9(7):784-90.

20. Zhou, YZ., Shen, XB., Shi, XQ., Lei, PY., Li, J., Wang, SP., et al. (2009). Analysis on causes and countermeasures of delay diagnosis of Pulmonary tuberculosis patients in north GuiZhou. Modern Prevent Med; 36: 4462-4. 